\title{
Value of fluorescein iridography in diagnosis of tumours of the iridociliary zone
}

\author{
A. F. BROVKINA AND A. G. CHICHUA \\ From the Department of Ophthalmo-oncology, Helmholtz Institute of Eye Diseases, Moscow
}

SUMMARY Fluorescein iridioangiography of 33 eyes with benign tumours and 22 eyes with malignant tumours is discussed. In cases of leiomyoma the tumour vessels have a distinct pattern, and fluorescence of the tumour tissue is mottled and short-lived. Malignant tumours are characterised by distortion of their vessels. Simultaneously the patches of fluorescence appear in the tissue of the tumour and lead to a diffuse confluence. Changes in the architecture of the vessels of the iris near the tumour and absence of fluorescence at the pupil margin indicate growth of the neoplasm.

At present local excision of tumours of the iris and ciliary body is commonly undertaken, but it is often difficult to be sure of the exact nature and extent of the neoplasm. The importance of fluorescein angiography in the diagnosis of choroidal tumours is well known (Sollom, 1968; Brovkina and Zinchenko, 1977), but this technique is rarely used in the diagnosis of tumours of the iris and the ciliary body. The literature on this subject is based on the examination of small groups of patients (Craandijk and Kerk, 1970; Sevel and Tobias, 1972; Wilensky and Holland, 1974; Freeman and Friedman, 1975; Brovkina et al., 1977).

The results of fluorescein angiography obtained in 55 patients with tumours of the iris and iridociliary region is the subject of the present paper.

\section{Patients and methods}

Fifty-five patients ( 22 males and 33 females) were examined. The age of the patients ranged from 16 to 62 (mean 45.9 years). In 38 patients $(69 \%)$ the tumour was situated both in the ciliary body and in the iris, in $15(27 \%)$ in the peripheral part of the iris only, and in 2 the pupillary portion of the iris was the site of the growth.

All the patients were treated surgically. In 52 cases local excision was performed, and 3 eyes were enucleated.

Benign tumours were found in 33 cases, 30 of which were leiomyomas, 2 were naevi, and 1 was a haemangioma. Twenty-two malignant tumours were

Address for reprints: A. F. Brovkina, Department of Ophthalmo-oncology, Helmholtz Institute of Eye Diseases, 14-19 Sadovo-Chernogrjazscaja Street, Moscow 103064, USSR found, including melanomas in 17 eyes, epitheliomas in 2 eyes, leiomyoblastomas in 2 eyes, and a prostatic metastasis in 1 eye.

The technique of fluorescein iridoangiography has been described elsewhere (Brovkina et al., 1977). $5 \mathrm{ml} 10 \%$ sodium fluorescein is injected into the antecubital vein. By means of a photo-slit lamp SLF (Carl Zeiss, Jena) with special filters photography is performed every 2 seconds, starting 5 seconds after the introduction of the dye and continuing up to 40 seconds. A control picture is taken 40 minutes later.

\section{Results}

Leiomyoma of the iris was the most frequent finding in our material. It occurred in 30 cases of $55(54.5 \%)$. In 7 cases the tumour was light brown or greyish brown and in 23 yellowish or pink. In 21 cases the tumour was situated in the iridociliary zone extending along the surface of the iris and producing ectopia uvea. In 8 eyes the tumour was localised to the iris root area without deforming the pupil. In 1 case a leiomyoma formed a solitary nodule at the margin of the pupil.

Angiographic findings in the eyes with leiomyoma were as follows. In 23 cases dotted staining of the tumour was observed. The dots were thought to be cross-sections of the tumour vessels, since they appeared and disappeared simultaneously with contrasting vessels of the iris.

In six eyes with non-pigmented and slightly pigmented leiomyomas a vascular network of the tumour formed a picture similar to the veins of a green leaf (Figs. 1 and 2). In 7 eyes with heavily pigmented tumours vessels were not seen. Nor were 


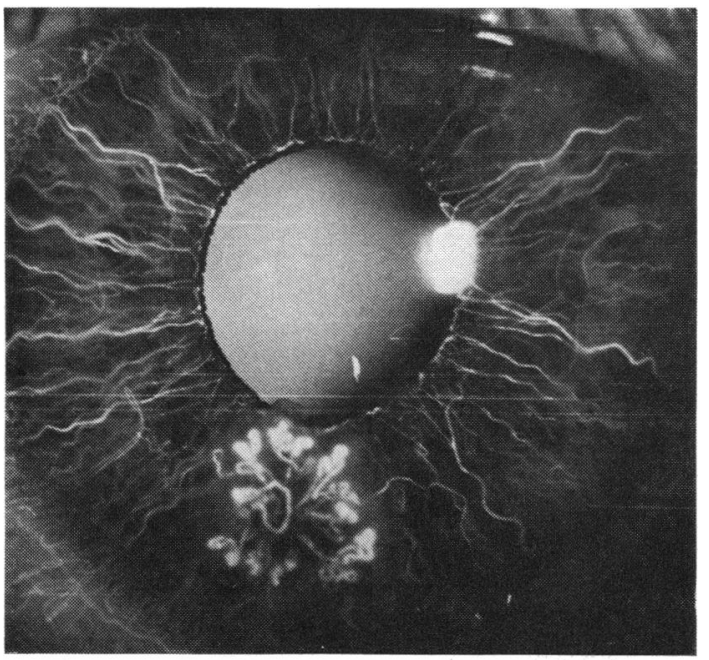

Fig. 1 Early phase fluorescein angiogram showing the blood vessel network of a leiomyoma

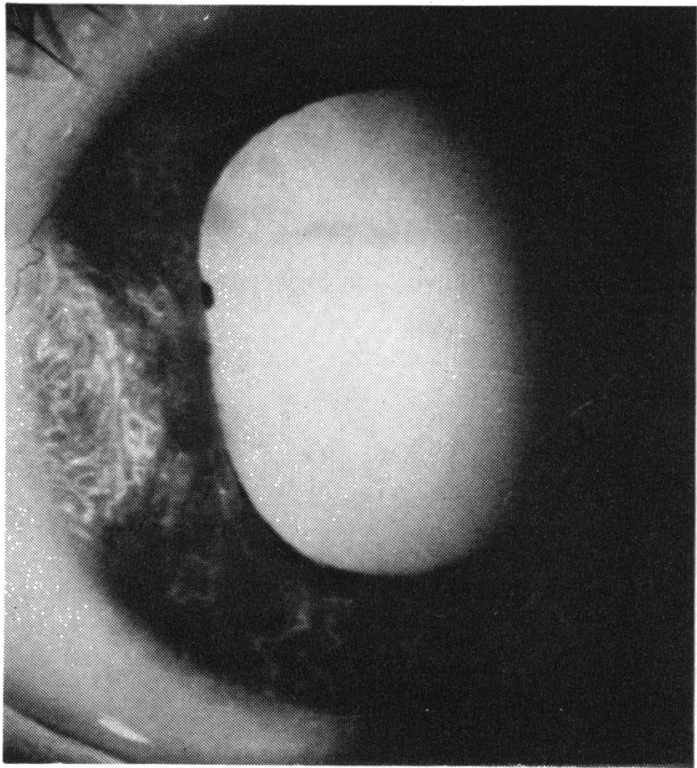

Fig. 2 Fluorescein angiogram showing 'veins of a green leaf' pattern

they seen in the tumour situated at the pupil margin, which resembled a jelly-like amorphous tissue through the slit lamp.

Mottled extravascular staining of the tumour was observed in 17 cases. The duration of the mottled fluorescence did not exceed 30 to 35 seconds. Not a single case of leiomyoma showed diffuse staining of the tumour tissue.
Fluorescence of the pupil margin round the whole circumference occurred in 4 cases with tumours localised to the iris root. In 23 cases fluorescence was seen only in the area of the pupil margin opposite the tumour, 16 of them showing a tumour within the iridociliary zone, 6 in the peripheral iris zone, and 1 in the pupillary zone. Complete absence of fluorescence of the pupil margin was found in 3 cases with a tumour localised in the iridociliary zone.

Second in frequency were melanomas. They occurred in 17 cases $(30.9 \%)$. In 10 cases the tumour was dark brown or black, in 4 light brown, and in 3 greyish pink. In 17 eyes the tumour was clinically localised to the iridociliary area. Histologically in 10 cases the tumour extended up to the pupil margin.

Iridoangiography in 4 cases resulted in fluorescence of the vessels of the iris and tumour vessels, which in all cases were distorted, shortened, and widened (Fig. 3). At first the superficial vessels of the tumour fluoresced, the deeper vessels showing fluorescence somewhat later. On the angiograms they were presented in the shape of circular, sharply outlined dots, which seemed to be the cross-sections of the vessels. Patches of fluorescence of the tumour tissue appeared simultaneously with fluorescence of its vessels. Rapid confluence of fluorescent zones of the tumour was characteristic of these cases, which created a picture of steady diffuse fluorescence of the total surface of the tumour. This lasted up to $\mathbf{4 0}$ minutes when the control photograph was taken.

Four cases displayed on the angiogram rather intense fluorescence of the iris round the tumour.

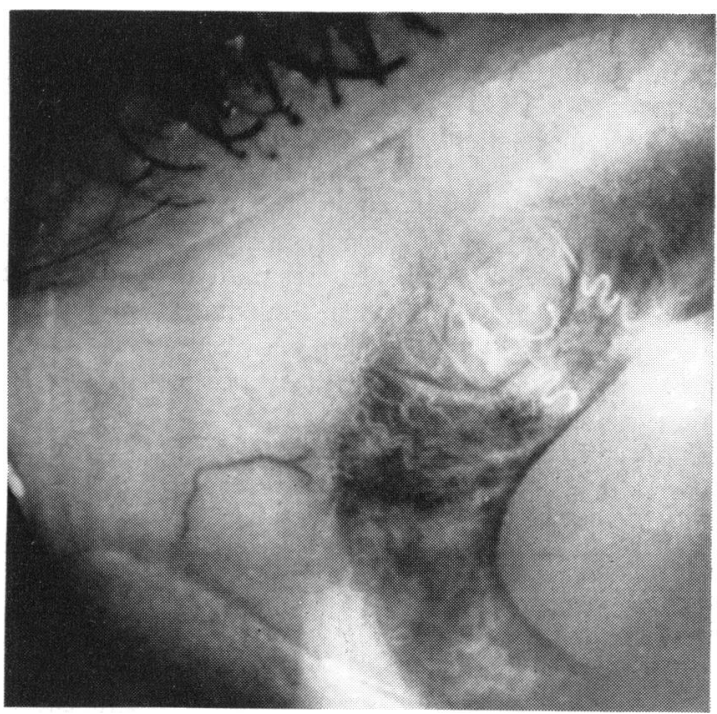

Fig. 3 Fluorescein iridoangiogram of a melanoma of the ciliary body 


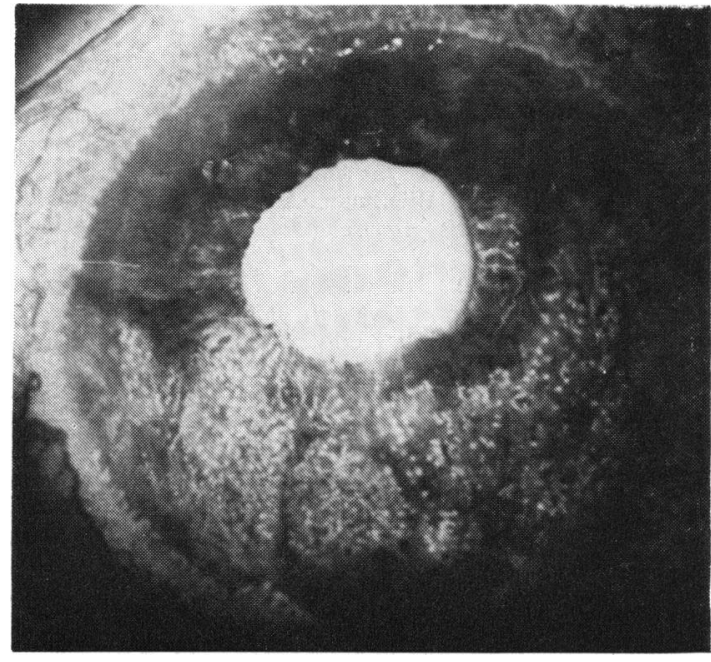

Fig. 4 Fluorescein iridoangiography of a metastatic carcinoma of the iris

In 12 cases noticeable changes were found in the vessels of the iris round the tumour. They showed distension and tortuosity. In 3 other cases an avascular zone in the iris round the tumour was observed. The pupil margin did not fluoresce in 14 cases.

Leiomyoblastoma occurred in 2 cases. Angiographic examination displayed fluorescence of the tumour vessels dispersed on the surface. Simultaneously there occurred mottled staining of the tumour itself, tending towards diffuse confluence. However, the fluorescence of the tumour did not last long, and it had practically disappeared by the end of the examination ( 40 seconds). There was no fluorescence of the pupil margin in the tumour zone.

Epithelioma of the ciliary body expanding into the iris was found in 2 eyes. In both cases the tumour was intensely pigmented, almost black. In 1 case the angiogram displayed uneven fluorescence of the iris round the tumour, which appeared after filling of the vessels of the iris with the dye.

There was 1 case of metastatic carcinoma of the iris. The tumour invaded the lower half of the iris and looked like a porous greyish mass. Iridoangiography displayed a deep change in vascular architecture of the iris, a considerable number of vessels in the tumour, with early mottled fluorescence of the tumour tissue. There was no fluorescence of the pupil margin (Fig. 4).

Haemangioma of the iris was situated in the lower temporal part of the pupil margin and looked like a pink bunch of grapes. Iridoangiography showed an unaltered net of fluorescent vessels of the iris. The pupil margin was intensely fluorescent except in the area occupied by the tumour. There was no fluorescence in the tumour.

Naevus of the iris is commoner than our material would suggest ( 2 eyes, $3.6 \%$ of all cases). This is accounted for by the fact that in this paper only histologically verified cases were analysed and local excision of a naevus is rarely performed. A naevus usually presented as a flat dark spot on the surface of the iris in the peripheral zone. Iridoangiography showed no changes in the vessels of the iris. The only exception was the zone of the naevus. In this zone there were no vessels or patches of pathological fluorescence. In both cases the fluorescence of the pupil margin was normal.

\section{Discussion}

Fluorescein angiography is useful in the diagnosis and differential diagnosis of tumours of the iris and the iridociliary zone. In some cases fluorescein photography shows the extent of the tumour better than other clinical methods.

One of the characteristic features of a growing tumour is the appearance of newly formed vessels which are filled with the dye at the same time as the iris vessels. In some cases cross-sections of the newly formed vessels are seen which look like circular, sharply outlined dots. In the course of the filling of the tumour vessels in leiomyomas a quarter of the cases observed produced the 'veins of a green leaf' pattern, which never presented in association with malignant tumours. In contrast, malignant tumours, especially melanomas, were characterised by a chaotic pattern of deformed vessels. The study of vessels in a tumour by angiography is feasible only in unpigmented and lightly pigmented tumours, as shown by Sevel and Tobias (1972), and Gass (1973).

Tumours are also characterised by fluorescein staining of their tissues. In cases of leiomyoma fluorescence is usually mottled, situated close to the vessels, and disappears within $\mathbf{4 0}$ seconds of injection of the dye. A significant feature of malignant tumours is a deeper diffuse staining. At first patches of tissue fluorescence appear. The intensity of fluorescence increases, zones of confluence then accrete and gradually diffuse staining develops across the whole tumour, which lasts for 30 to 40 minutes. This pattern is characteristic of melamoma (Amalrik and Rebiere, 1971; Wilensky and Holland, 1974; Demeler and Domarus, 1976). Rapidly developing intense fluorescence of tumour tissue suggests patency of newly formed vessels, permitting leakage of dye.

There is no fluorescence of the iris tissue in normal eyes (Harris et al., 1972). The angiogram shows only the vascular network of the iris and even fluorescence 
of the pupil margin. In eyes with melanomas of the iris pronounced staining of the iris tissue round the tumour was seen. Expansion of the tumour leads to a disturbance of the circulation at the pupil margin, which is shown on the iridoangiogram by absence of fluorescence. In eyes with melanoma of the iris fluorescence of the pupil margin did not occur at all. In cases of leiomyoma and haemangioma fluorescence was observed only in the zone opposite the localisation of the tumour.

In all our cases fluorescein iridoangiography revealed noticeable changes in the vessels of the iris round the tumour such as displacement, distension, and tortuosity.

\section{References}

Amalrik, P., and Rebiere, P. (1971). Nouvelles indications de l'angiographie fluoresceinique du segment antérieur de l'oeil. Annales d'Oculistique, 204, 595-609.

Brovkina, A. F., Chichua, A. G., and Balishanskaya, T. I. (1977). Fliuorestsentnaia angiografiia $v$ diagnostike opukholei iridotsiliarnoi zony. Vestnik Oftal' mologii, 1, 68-73.

Brovkina, A. F., and Zinchenko, L. I. (1977). In The Problems of Clinical Ophthalmology, p. 183. Alma-Ata.

Demeler, U., and Domarus, D. (1976). Klinik, fluoreszenzangiographie und histologie eines ringmelanoms der iris. Klinische Monatsblätter für Augenheilkunde, 168, 387-395.

Freeman, T. R., and Friedman, A. H. (1975). Metastatic carcinoma of the iris. American Journal of Ophthalmology, 80, 947-952.

Gass, J. D. (1973). Iris abscess simulating malignant melanoma. Archives of Ophthalmology, 90, 300-302.

Craandijk, A., and Aan de Kerk, A. L. (1970). Fluorescence angiography of the iris. British Journal of Ophthalmology, 54, 229-232.

Harris, L. S., Toyufuku, H., and Shimmyo, M. (1972). Fluorescein iris angiography in the albino rabbit. Archives of Ophthalmology, 88, 193-195.

Sevel, D., and Tobias, B. (1972). The value of fluorescein iridography with leiomyoma of the iris. American Journal of Ophthalmology, 74, 475-478.

Sollom, A. W. (1968). Fluorescence in malignant melanoma of the choroid. Ophthalmologica, 156, 117-123.

Wilensky, J. T.; and Holland, M. G. (1974). A pigmented tumour of the ciliary body. Archives of Ophthalmology, 92, 219-220. 